\title{
FATORES QUE INFLUENCIAM A SEGURANÇA DO PACIENTE EM SERVIÇOS DE URGÊNCIA E EMERGÊENCIA: REVISÃO INTEGRATIVA
}

\author{
FACTORS THAT INFLUENCE PATIENT SAFETY \\ IN URGENCY AND EMERGENCY SERVICES: \\ INTEGRATIVE REVIEW
}

\section{FACTORES QUE INFLUYEN EN LA SEGURIDAD DEL PACIENTE EN LOS SERVICIOS DE URGENCIA Y EMERGENCIA: REVISIÓN INTEGRADORA}

\author{
Eloyne Tavares da Silva ${ }^{1}$ \\ Laura Misue Matsuda ${ }^{2}$ \\ Gabriela Machado Ezaias Paulino ${ }^{3}$ \\ Nadia Raquel Suzini Camillo ${ }^{4}$ \\ Ana Carolina Simões 5 \\ Andressa Martins Dias Ferreira ${ }^{6}$
}

Como citar este artigo: Silva ET, Matsuda LM, Paulino GME, Camillo NRS, Simões AC, Ferreira AMD. Fatores que influenciam a segurana do paciente em serviços de urgência e emergência: revisão integrativa. Rev baiana de enferm. 2019;33:e33408.

Objetivo: identificar evidências disponíveis na literatura científica acerca de fatores que interferem na segurança do paciente em serviços de urgência e emergência. Método: revisão integrativa da literatura realizada conforme as etapas: identificação do tema e questão norteadora; estabelecimento de critérios de inclusão e exclusão para os estudos; busca na literatura de estudos primários; avaliação da amostra de estudos incluídos na revisão, com extração de dados; interpretação dos resultados e apresentação. Resultados: foram selecionadas 1.449 publicações que, após aplicação dos critérios de inclusão e leitura detalhada, compuseram um conjunto de oito publicações. Nos textos foram identificados diferentes aspectos que influenciam a segurança do paciente nos serviços de urgência e emergência, os quais foram categorizados de acordo com a técnica análise de conteúdo: fatores organizacionais; falhas na comunicação da equipe e fragilidades no processo de medicação. Conclusão: múltiplos fatores interferem na segurança do paciente no ambiente emergencial, destacando-se o moderado número de pacientes e a sobrecarga de trabalho.

Descritores: Segurança do Paciente. Serviço Hospitalar de Emergência. Enfermagem.

Objective: to identify the available evidence in the scientific literature about factors that interfere in patient safety in urgency and emergency services. Method: integrative literature review performed according to the following steps: identification of the theme and guiding question; establishment of inclusion and exclusion criteria for the study; search in the literature for primary studies; evaluation of the sample of studies included in the review, with data

\footnotetext{
Enfermeira. Pesquisadora independente. Maringá, Paraná, Brasil. http://orcid.org/0000-0002-06 I5-4604.eloyneh.tavares@gmail.com

Enfermeira. Doutora em Enfermaem Fundamental. Professora da Universidade Estadual de Maringá. Maringá, Paraná, Brasil. http://orcid.org/0000-000 I-6075-665X.

Enfermeira. Mestre em Enfermagem Fundamental. Professora da Universidade Estadual de Maringá. Londrina, Paraná, Brasil. http://orcid.org/0000-0002-0697-5795.

Enfermeira. Mestre em Enfermagem Fundamental. Pesquisadora Independente. Maringá, Paraná, Brasil. http://orcid.org/0000-000 I-5 I05-7806.

Enfermeira. Professora da Universidade Estadual de Maringá. Londrina, Paraná, Brasil. http://orcid.org/0000-000 I-6075-665X.

Enfermeira. Professora da Universidade Estadual de Maringá. Londrina, Paraná, Brasil. http://orcid.org/0000-0002-8020-9773.
} 
extraction; interpretation of results and presentation. Results: 1,449 publications were selected, which, after applying the inclusion criteria and detailed reading, comprised a set of eight publications. The texts revealed different aspects that influence patient safety in urgency and emergency services, categorized according to the content analysis technique: organizational factors; team communication failures and weaknesses in the medication process. Conclusion: multiple factors interfere in patient safety in the emergency environment, highlighting the moderate number of patients and work overload.

Descriptors: Patient Safety. Emergency Service, Hospital. Nursing.

Objetivo: identificar la evidencia disponible en la literatura científica acerca de los factores que interfieren en la seguridad del paciente en los servicios de urgencia y emergencia. Método: revisión integradora de la literatura realizada de acuerdo con los siguientes pasos: identificación del tema y de la pregunta orientadora; establecimiento de los criterios de inclusión y exclusión para el estudio; búsqueda en la literatura de los estudios primarios; evaluación de la muestra de los estudios incluidos en la revisión, con extracción de datos; interpretación de los resultados y presentación. Resultados: se seleccionaron 1.449 publicaciones que, después de aplicar los criterios de inclusión y lectura detallada, compusieron un conjunto de ocho publicaciones. En los textos, fueron identificados diferentes aspectos que influyen en la seguridad del paciente en los servicios de urgencia y emergencia, que fueron clasificados de acuerdo con la técnica de análisis de contenido: factores organizativos; comunicación falla del equipo y debilidades en el proceso de medicación. Conclusión: múltiples factores interfieren en la seguridad de los pacientes en situación de urgencia, destacando el número moderado de pacientes y la sobrecarga de trabajo.

Descriptores: Seguridad del Paciente. Servicio de Urgencia en Hospital. Enfermería.

\section{Introdução}

A segurança do paciente é apreendida atualmente pelos profissionais da área, como tema relevante para as práticas em saúde, no sentido de evitar, prevenir e/ou mitigar riscos e eventos adversos (EA) em pacientes de todos os níveis de atenção ${ }^{(1)}$. Desse modo, organizações mundiais de atenção à saúde têm abordado a segurança como componente direto da qualidade assistencial ${ }^{(2)}$.

De acordo com a Organização Mundial da Saúde (OMS), milhões de pacientes sofrem anualmente lesões incapacitantes que resultam de erros no atendimento à saúde ${ }^{(3)}$. Em países desenvolvidos, um em cada dez pacientes sofre algum tipo de evento adverso (EA) em ambientes hospitalares $^{(3-4)}$. No Brasil, o Ministério da Saúde (MS) menciona que, de cada 10\% dos EA registrados, 50\% seriam evitáveis. Dentre os eventos mais frequentemente notificados, constam as complicações clínicas, cirúrgicas, quedas do leito, erros de medicação e infecções ${ }^{(5)}$.

Eventos adversos são frequentemente associados ao erro humano individual ${ }^{(6)}$. Contudo, no cenário de urgências/emergências devem-se considerar também como fatores desencadeantes de erros as condições de trabalho, como: superlotação de pacientes, alta carga de trabalho, interrupções do processo assistencial e gerenciamento simultâneo de múltiplas tarefas ${ }^{(7-9)}$, as quais aumentam a complexidade das atividades desenvolvidas cujas situações predispõem a riscos de eventos adversos ${ }^{(10)}$.

Com o intuito de ampliar as ações para a promoção da segurança do paciente no Brasil, foi estabelecido, em 2013, o Programa Nacional de Segurança do Paciente (PNSP), mediante a Portaria n. 529/2013 do MS e da Resolução da Diretoria Colegiada (RDC) n. 36/2013, as quais instituíram ações para a segurança do paciente nos serviços de saúde, por meio da implantação de protocolos, de Núcleos de Segurança do Paciente (NSP) e de sistemas de notificações de $\mathrm{EA}^{(3)}$.

Nos serviços de urgência e emergência, há situações que exigem dos profissionais rapidez para o raciocínio clínico e a tomada de decisões seguras $^{(11)}$. Esses eventos, atrelados à rigidez dos protocolos assistenciais, expõem os profissionais a situações estressoras que podem resultar em déficits na qualidade do cuidado emergencial ${ }^{(12)}$. Nesse aspecto, a literatura aponta que 50\% dos eventos adversos seriam evitáveis. Dentre aqueles mais frequentemente ocorridos em unidades de emergência, esses são passíveis de prevenção ${ }^{(13)}$. 
Considerando a importância de estudos de revisão sistemática, que são instrumentos importantes no campo da saúde, por sintetizar as pesquisas sobre determinado tema e ser um método relevante para a prática baseada em evidências $^{(14)}$, teve-se como questão norteadora: "Que fatores influenciam a segurança do paciente em serviços de urgência e emergência?"

Este estudo tem como objetivo identificar evidências disponíveis na literatura cientifica acerca de fatores que interferem na segurança do paciente em serviços de urgência e emergência.

\section{Método}

Revisão Integrativa da Literatura (RIL) realizada conforme as etapas propostas por estudo ${ }^{(15)}$ que apresenta método de pesquisa para a incorporação de evidências na saúde e na enfermagem, quais sejam: identificação do tema e da questão norteadora, estabelecimento de critérios de inclusão e exclusão para os estudos, busca na literatura de estudos primários, avaliação da amostra de estudos incluídos na revisão com extração de dados, interpretação dos resultados e apresentação da RIL. Para a elaboração da questão norteadora, utilizou-se a estratégia PCC, acrônimo para os termos "population", "concept" e "context"

As buscas por publicações foram realizadas nas bases de dados Scientific Electronic Library Online (SciELO), Literatura Latina-Americana e do Caribe em Ciências da Saúde (LILACS), Library of Medicine National Institute of Health (PubMED), SciVerse Scopus (Scopus), Cumulative Index to Nursing and Allied Health Literature (CINAHL) e Web of Science, por meio da combinação dos descritores controlados, selecionados do banco de Descritores em Ciências da Saúde (DeCS), Medical Subject Headings (MeSH Database) e Cumulative Index to Nursing and Allied Health Literature (CINAHL), quais sejam: "segurança do paciente", "serviço hospitalar de emergência", "patient safety" e "emergency service". Em todas as bases, os descritores foram combinados com o operador boleano " $A N D$ ".

Como critérios de inclusão estebeleceram-se: estudo primário, com abordagem de fatores que influenciam a segurança do paciente no contexto assistencial dos serviços de urgência e emergência, publicados nos idiomas português, espanhol ou inglês, no período de 2013 a 2017 (cinco anos). Foram excluídos os estudos primários que obtiveram menos de 80\% das afirmativas no checklist proposto por Caldwell, Henshaw e Taylor, usado para avaliar o trabalho qualitativo e quantitativo em termo de estrutura produzida, que utiliza recursos comuns a ambas as metodologias de pesquisa, bem como as que diferem ${ }^{(17)}$.

Foram excluídos artigos que pertenciam às seguintes modalidades: artigos de revisão de literatura, relatos de experiência, artigos reflexivos, editorial, estudo de caso e artigos repetidos. Após a leitura de títulos e resumos, foram excluídas as publicações que não abordavam o tema do estudo e/ou apresentavam-se em duplicidade. Ademais, os artigos selecionados foram analisados pelo instrumento de Checklist referido ${ }^{(17)}$. Foram excluídos estudos primários que obtiveram menos de $80 \%$ de respostas afirmativas no instrumento.

Para extração dos dados, utilizou-se um instrumento adaptado e validado para o Brasil $^{(18)}$. Em seguida, os dados foram categorizados de acordo com a técnica análise de conteúdo temática ${ }^{(19)}$, constituída das seguintes etapas: pré-análise, na qual foi realizada a leitura flutuante e familiarização com os textos selecionados; exploração do material, com identificação de núcleos de sentido e categorização, conforme similaridades dos achados; e tratamento e inferência/interpretação dos resultados, de modo a responder à questão de pesquisa e ao objetivo do estudo.

No que se refere aos aspectos éticos, houve dispensa da submissão ao Comitê Permanente de Ética em Pesquisas Envolvendo Seres Humanos (COPEP).

\section{Resultados}

Foram identificadas 1.449 publicações que, após a aplicação dos critérios de inclusão e exclusão, leitura detalhada dos títulos, resumos e textos na íntegra, compuseram um conjunto de oito publicações, conforme se vê na Figura 1. 
Figura 1 - Fluxo da seleção dos estudos primários de acordo com as bases de dados

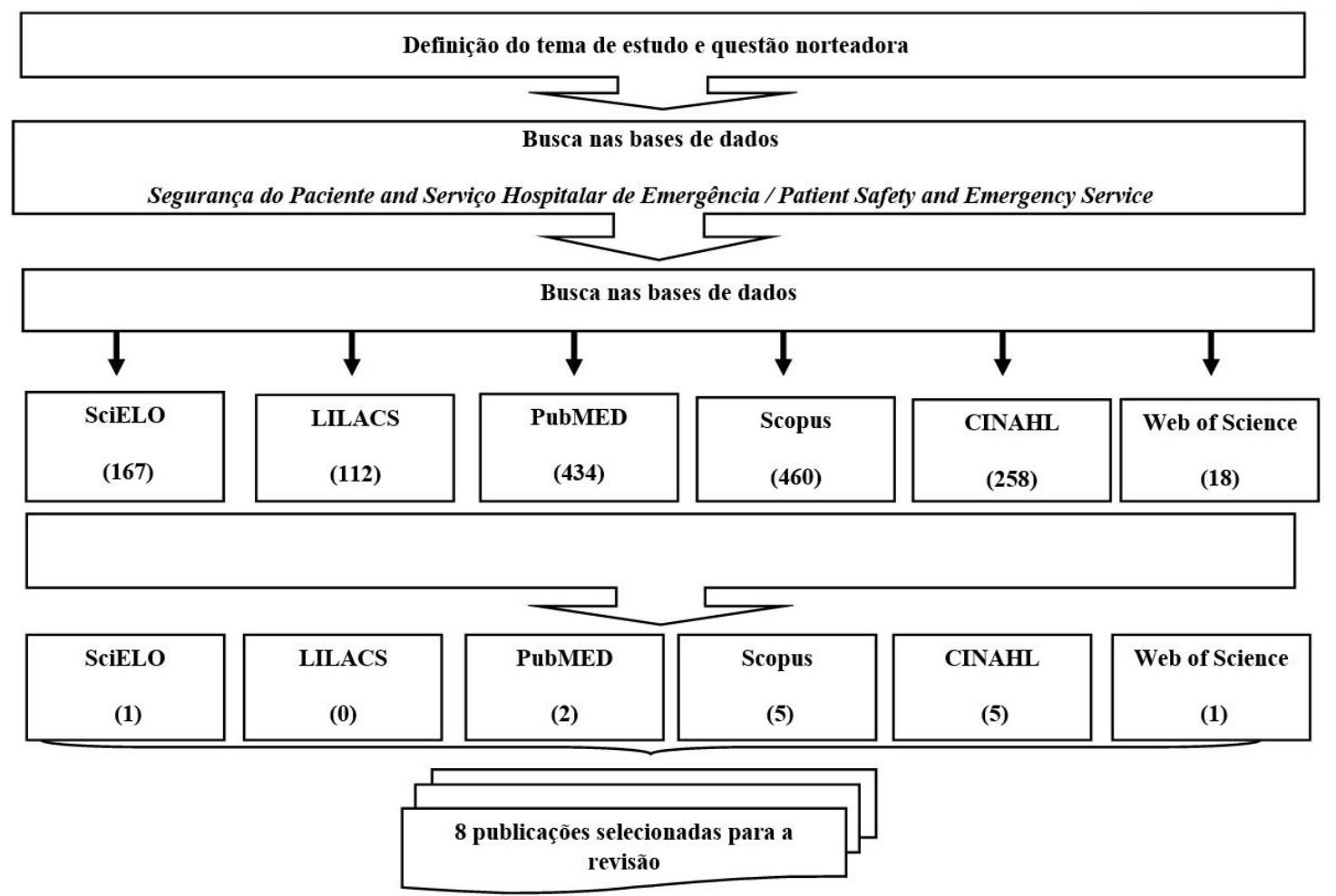

Fonte: Elabração própria.

No que se refere ao idioma, a maioria dos $\operatorname{artigos}(n=5)$ foi publicada em países com predomínio da língua inglesa, fato que denota a escassez de publicações referentes ao tema no contexto latino-americano. Outro dado a ser destacado é que, dentre os artigos selecionados, cinco encontravam-se na base de dados CINAHL.
Quanto ao tipo de pesquisa, verificou-se que seis eram de abordagem quantitativa, quatro eram qualitativas e nenhuma utilizou a abordagem quali-quantitativa ou de métodos mistos. No Quadro 1 constam as principais informações extraídas dos artigos.

Quadro 1 - Síntese dos estudos primários incluídos na RIL ( $\mathrm{n}=8$ )

(continua)

\begin{tabular}{|c|c|c|c|c|}
\hline $\begin{array}{c}\text { Ano/País/ } \\
\text { Base de } \\
\text { dados }\end{array}$ & $\begin{array}{c}\text { Título do } \\
\text { artigo }\end{array}$ & Objetivo & Principais resultados & Conclusões \\
\hline $\begin{array}{l}2014 \\
\text { Bélgica } \\
\text { CINAHL }\end{array}$ & $\begin{array}{l}\text { The number } \\
\text { of patients } \\
\text { simultaneously } \\
\text { present at the } \\
\text { emergency } \\
\text { department as } \\
\text { an indicator of } \\
\text { unsafe waiting } \\
\text { times: a receiver } \\
\text { operated } \\
\text { curve-based } \\
\text { evaluation }\end{array}$ & $\begin{array}{l}\text { Investigar se a } \\
\text { quantidade de } \\
\text { pacientes em } \\
\text { espera no setor } \\
\text { de emergência } \\
\text { e o tempo de } \\
\text { espera para } \\
\text { atendimento é } \\
\text { um indicador de } \\
\text { insegurança e } \\
\text { quais condutas } \\
\text { hospitalares } \\
\text { podem otimizar } \\
\text { o fluxo do } \\
\text { atendimento. }\end{array}$ & $\begin{array}{l}\text { Os resultados foram } \\
\text { avaliados de acordo com } \\
\text { o Emergency Severity Index } \\
\text { (ESI), o qual indica como } \\
\text { tempo de espera máximo } \\
\text { para pacientes classificados } \\
\text { com os escores ESI } 1 \text { e ESI } 2 \\
\text { respectivamente, } 10 \text { minutos } \\
\text { e } 30 \text { minutos. A média de } \\
\text { tempo de espera foi de } \\
5 \text { minutos para pacientes } \\
\text { de maior gravidade e de } \\
12 \text { minutos para pacientes } \\
\text { menos graves. }\end{array}$ & $\begin{array}{l}\text { O número de } \\
\text { pacientes nas } \\
\text { unidades de } \\
\text { emergência é } \\
\text { um indicador } \\
\text { moderado de } \\
\text { insegurança; } \\
\text { iniciativas futuras } \\
\text { para melhorar } \\
\text { o tempo de } \\
\text { espera seguro } \\
\text { não devem } \\
\text { concentrar-se na } \\
\text { taxa de ocupação, } \\
\text { mas incluir outros } \\
\text { fatores que } \\
\text { afetam o tempo } \\
\text { de espera. }\end{array}$ \\
\hline
\end{tabular}


Quadro 1 - Síntese dos estudos primários incluídos na RIL ( $\mathrm{n}=8)$

\begin{tabular}{|c|c|c|c|c|}
\hline $\begin{array}{c}\text { Ano/País/ } \\
\text { Base de } \\
\text { dados }\end{array}$ & $\begin{array}{l}\text { Título do } \\
\text { artigo }\end{array}$ & Objetivo & Principais resultados & Conclusões \\
\hline $\begin{array}{l}2015 \\
\text { Suíça } \\
\text { CINAHL }\end{array}$ & $\begin{array}{l}\text { Contributing } \\
\text { factors to errors } \\
\text { in Swedish } \\
\text { emergency } \\
\text { departments }\end{array}$ & $\begin{array}{l}\text { Descrever fatores } \\
\text { que contribuem } \\
\text { para a ocorrência } \\
\text { de erros em } \\
\text { serviços de } \\
\text { emergência na } \\
\text { Suécia. }\end{array}$ & $\begin{array}{l}\text { Constataram-se erros no } \\
\text { planejamento e/ou na } \\
\text { execução da assistência, } \\
\text { falta de supervisão, falhas } \\
\text { na rotina de trabalho, erros } \\
\text { de comunicação entre } \\
\text { profissionais, falta de trabalho } \\
\text { em equipe, alta carga de } \\
\text { trabalho e dificuldade na } \\
\text { comunicação com o paciente. } \\
\text { Além disso, devido à falta } \\
\text { de apoio à decisão e rotina } \\
\text { deficiente, há erros na } \\
\text { triagem, dificuldade de fluxo } \\
\text { com os sistemas externos, } \\
\text { acesso e administração. }\end{array}$ & $\begin{array}{l}\text { Os erros em } \\
\text { serviços de } \\
\text { emergência } \\
\text { têm natureza } \\
\text { multifatorial, } \\
\text { incluindo falhas } \\
\text { organizacionais } \\
\text { e no trabalho } \\
\text { em equipe. Os } \\
\text { fatores humanos } \\
\text { relacionaram-se } \\
\text { principalmente } \\
\text { aos procedimentos } \\
\text { diagnósticos e } \\
\text { comportamento } \\
\text { humano. }\end{array}$ \\
\hline $\begin{array}{l}2014 \\
\text { Canadá } \\
\text { CINAHL }\end{array}$ & $\begin{array}{l}\text { Detection and } \\
\text { correction of } \\
\text { prescription } \\
\text { errors by an } \\
\text { emergency } \\
\text { department } \\
\text { pharmacy }_{\text {service }^{(22)}}\end{array}$ & $\begin{array}{l}\text { Descrever a } \\
\text { frequência e o } \\
\text { tipo de erros } \\
\text { de prescrição } \\
\text { detectados por } \\
\text { farmacêuticos } \\
\text { em serviços de } \\
\text { emergência; e } \\
\text { determinar a } \\
\text { proporção de } \\
\text { erros evitáveis e os } \\
\text { fatores associados a } \\
\text { erros de prescrição. }\end{array}$ & $\begin{array}{l}\text { Foram identificados erros } \\
\text { em } 3,2 \% \text { das prescrições. } \\
\text { Dentre os tipos de erros, } \\
\text { destacaram-se: erros na } \\
\text { dosagem }(28,3 \%) \text {, prescrição } \\
\text { incompleta }(27,3 \%) ; \\
\text { frequência errada }(15,2 \%) ; \\
\text { droga errada }(11,1 \%) ; \text { via } \\
\text { errada (1,0\%) e outros } \\
\text { (17,2\%). Os farmacêuticos } \\
\text { corrigiram a maioria das } \\
\text { prescrições com erros. }\end{array}$ & $\begin{array}{l}\text { Erros são mais } \\
\text { prováveis } \\
\text { de serem } \\
\text { identificados em } \\
\text { prescrições de } \\
\text { pacientes mais } \\
\text { velhos, naqueles } \\
\text { com múltiplas } \\
\text { medicações e/ou } \\
\text { prescritas por } \\
\text { residentes de } \\
\text { emergência. }\end{array}$ \\
\hline $\begin{array}{l}2014 \\
\text { Austrália } \\
\text { CINAHL }\end{array}$ & $\begin{array}{l}\text { Complexities } \\
\text { of medicines } \\
\text { safety: } \\
\text { communicating } \\
\text { about } \\
\text { managing } \\
\text { medicines } \\
\text { at transition } \\
\text { points of } \\
\text { care across } \\
\text { emergency } \\
\text { departments } \\
\text { and medical } \\
\text { wards }\end{array}$ & $\begin{array}{l}\text { Explorar como } \\
\text { profissionais de } \\
\text { saúde, pacientes } \\
\text { e familiares } \\
\text { comunicam-se } \\
\text { acerca da gestão } \\
\text { de medicamentos } \\
\text { nos pontos de } \\
\text { transição de } \\
\text { cuidados em dois } \\
\text { hospitais públicos } \\
\text { australianos. }\end{array}$ & $\begin{array}{l}\text { Quatro categorias: (1) } \\
\text { Ambiente Contextual } \\
\text { de Atenção: ambiente } \\
\text { desorganizado e constantes } \\
\text { interrupções na Unidade } \\
\text { de Emergência afetaram } \\
\text { a comunicação entre } \\
\text { paciente/família e equipe; } \\
\text { (2) Responsabilidades do } \\
\text { Cuidado: pouca atenção } \\
\text { aos pacientes devido aos } \\
\text { atendimentos de emergência, } \\
\text { atraso e/ou não administração } \\
\text { dos medicamentos ou } \\
\text { autoadministração sem } \\
\text { conhecimento da equipe; } \\
\text { (3) Conscientização } \\
\text { sobre responsabilidade } \\
\text { pela segurança: falta de } \\
\text { informações sobre uso } \\
\text { de medicamentos nos } \\
\text { momentos da asssistência; (4) } \\
\text { Comunicação Interprofissional: } \\
\text { na transição do cuidado, } \\
\text { as informações não eram } \\
\text { transmitidas com precisão. }\end{array}$ & $\begin{array}{l}\text { Os erros de } \\
\text { medicamentos } \\
\text { são comuns } \\
\text { em pontos } \\
\text { de transição } \\
\text { de cuidados. } \\
\text { A gestão de } \\
\text { medicamentos } \\
\text { nos pontos de } \\
\text { transição envolve } \\
\text { uma complexa } \\
\text { interação de } \\
\text { recursos. Essa } \\
\text { interação infiltrou- } \\
\text {-se em diversos } \\
\text { ambientes e } \\
\text { afetou tanto o } \\
\text { atendimento ao } \\
\text { paciente como } \\
\text { a capacidade de } \\
\text { comunicação } \\
\text { entre os } \\
\text { profissionais de } \\
\text { saúde. }\end{array}$ \\
\hline
\end{tabular}


Quadro 1 - Síntese dos estudos primários incluídos na RIL (n=8)

\begin{tabular}{|c|c|c|c|c|}
\hline $\begin{array}{c}\text { Ano/País/ } \\
\text { Base de } \\
\text { dados }\end{array}$ & $\begin{array}{l}\text { Título do } \\
\text { artigo }\end{array}$ & Objetivo & Principais resultados & Conclusões \\
\hline $\begin{array}{l}2014 \\
\text { Brasil } \\
\text { PubMed }\end{array}$ & $\begin{array}{l}\text { Incidentes: } \\
\text { instrumento de } \\
\text { gerenciamento } \\
\text { da assistência } \\
\text { para a } \\
\text { segurança } \\
\text { do paciente } \\
\text { em pronto } \\
\text { socorro }^{(24)}\end{array}$ & $\begin{array}{l}\text { Caracterizar } \\
\text { os incidentes } \\
\text { advindos do } \\
\text { cuidado à saúde, } \\
\text { ocorridos no } \\
\text { Pronto Socorro } \\
\text { de um hospital } \\
\text { universitário } \\
\text { pertencente à } \\
\text { Rede de Hospitais } \\
\text { Sentinela. }\end{array}$ & $\begin{array}{l}\text { Detectados } 75 \text { incidentes } \\
\text { dos quais } 38,7 \% \text { ( } 29 \text { ) } \\
\text { relacionavam-se à } \\
\text { organização do serviço } \\
\text { (falhas nas anotações, } \\
\text { previsão e provisão de } \\
\text { materiais e evasão do } \\
\text { paciente) e } 61,3 \% \text { (46) } \\
\text { referiam-se ao cuidado } \\
\text { (omissão de cuidado, erro } \\
\text { de medicação, falta de } \\
\text { humanização, infecções, } \\
\text { quedas e falhas em } \\
\text { procedimentos/técnicas). }\end{array}$ & $\begin{array}{l}\text { Constatou-se } \\
\text { deficiência nos } \\
\text { registros quanto } \\
\text { às consequências } \\
\text { dos incidentes } \\
\text { para os pacientes, } \\
\text { profissionais } \\
\text { e instituição } \\
\text { que, aliada à } \\
\text { subnotificação, } \\
\text { dificultaram a } \\
\text { tomada de decisão } \\
\text { e a elaboração } \\
\text { de estratégias } \\
\text { preventivas e de } \\
\text { controle. }\end{array}$ \\
\hline $\begin{array}{l}2015 \\
\text { Espanha } \\
\text { SciELO }\end{array}$ & $\begin{array}{l}\text { Estudio } \\
\text { Regional de } \\
\text { Incidentes } \\
\text { Derivados de } \\
\text { la Atención } \\
\text { (ERIDA) em } \\
\text { Urgencias }^{(25)}\end{array}$ & $\begin{array}{l}\text { Avaliar os } \\
\text { incidentes de } \\
\text { segurança do } \\
\text { paciente em } \\
\text { serviços de } \\
\text { urgências da } \\
\text { região }\end{array}$ & $\begin{array}{l}\text { Observou-se ao menos um } \\
\text { incidente em } 47 \text { (11,95\%) } \\
\text { pacientes e, em 3, verificou--se } \\
\text { a ocorrência de dois incidentes. } \\
\text { Dentre os fatores causais } \\
\text { constavam: erros de medicação } \\
\text { (reações adversas, medicação } \\
\text { errada, frequência incorreta e } \\
\text { falta de adesão ao tratamento), } \\
\text { falhas de comunicação (médico- } \\
\text {-paciente, enfermeiro- } \\
\text {-paciente e enfermeiro- } \\
\text {-médico), falhas na gestão } \\
\text { (erros na identificação do } \\
\text { paciente e espera prolongada), } \\
\text { falhas no diagnóstico (erro } \\
\text { diagnóstico e atraso), falhas no } \\
\text { cuidado (técnica inadequada } \\
\text { e manutenção inadequada de } \\
\text { cateteres). }\end{array}$ & $\begin{array}{l}\text { A maioria dos } \\
\text { erros não eram } \\
\text { potencialmente } \\
\text { fatais, mas as } \\
\text { causas que } \\
\text { sustentaram } \\
\text { esses erros eram } \\
\text { variadas. }\end{array}$ \\
\hline $\begin{array}{l}2016 \\
\text { Austrália } \\
\text { Scopus }\end{array}$ & $\begin{array}{l}\text { Associations } \\
\text { of work } \\
\text { characteristics, } \\
\text { employee } \\
\text { strain and } \\
\text { self-perceived } \\
\text { quality of care } \\
\text { in Emergency } \\
\text { Departments: A } \\
\text { cross-sectional } \\
\text { study }\end{array}$ & $\begin{array}{l}\text { Investigar } \\
\text { simultaneamente } \\
\text { as associações } \\
\text { entre múltiplas } \\
\text { características } \\
\text { contextuais do } \\
\text { trabalho das } \\
\text { unidades de } \\
\text { emergência, } \\
\text { bem-estar dos } \\
\text { funcionários e } \\
\text { qualidade dos } \\
\text { cuidados. }\end{array}$ & $\begin{array}{l}\text { Exaustão emocional e } \\
\text { irritação dos profissionais } \\
\text { apresentaram alta correlação } \\
\text { com a qualidade de cuidado } \\
\text { autopercebida. Após ajuste } \\
\text { para o tipo de contrato, } \\
\text { o aumento da exaustão } \\
\text { correlacionou--se com } \\
\text { avaliações mais baixas da } \\
\text { qualidade do cuidado [B=0,30 } \\
\text { (95\% IC 0,57; 0,03)]; nível alto } \\
\text { de irritação relacionou-se com } \\
\text { a diminuição da percepção } \\
\text { da qualidade do atendimento } \\
\text { [B=0,37 (IC 95\% 0,63; 0,11)]. } \\
\text { As condições adversas de } \\
\text { trabalho associaram-se com } \\
\text { baixa percepção da qualidade } \\
\text { do cuidado. }\end{array}$ & $\begin{array}{l}\text { Cuidados confiáveis } \\
\text { em unidade } \\
\text { de emergência } \\
\text { dependem da } \\
\text { percepção do } \\
\text { pessoal sobre } \\
\text { o ambiente de } \\
\text { trabalho e do bem- } \\
\text {-estar mental da } \\
\text { equipe. O apoio } \\
\text { da supervisão, o } \\
\text { número adequado } \\
\text { de pessoal e a } \\
\text { redução da tensão, } \\
\text { são pontos de } \\
\text { partida importantes } \\
\text { para os esforços de } \\
\text { melhoria da quali- } \\
\text { dade do cuidado }\end{array}$ \\
\hline
\end{tabular}


Quadro 1 - Síntese dos estudos primários incluídos na RIL ( $\mathrm{n}=8)$ (conclusão)

\begin{tabular}{|c|c|c|c|c|}
\hline $\begin{array}{c}\text { Ano/País/ } \\
\text { Base de } \\
\text { dados }\end{array}$ & $\begin{array}{l}\text { Título do } \\
\text { artigo }\end{array}$ & Objetivo & Principais resultados & Conclusões \\
\hline $\begin{array}{l}2017 \\
\text { Suécia } \\
\text { Web of } \\
\text { Science }\end{array}$ & $\begin{array}{l}\text { Physicians' } \\
\text { and nurses' } \\
\text { perceptions of } \\
\text { patient safety } \\
\text { risks in the } \\
\text { emergency } \\
\text { department }\end{array}$ & $\begin{array}{l}\text { Descrever a } \\
\text { percepção } \\
\text { dos médicos } \\
\text { e enfermeiros } \\
\text { de unidades de } \\
\text { emergência a } \\
\text { respeito de riscos } \\
\text { para a segurança } \\
\text { do paciente. }\end{array}$ & $\begin{array}{l}\text { Categorias: (1) alta carga } \\
\text { de trabalho - demanda } \\
\text { excessiva de pacientes e } \\
\text { atendimento simultâneo } \\
\text { de mais de um paciente } \\
\text { em estado grave; (2) falta } \\
\text { de controle - multitarefas } \\
\text { e interrupções durante a } \\
\text { execução dos cuidados, } \\
\text { profissionais com pouca } \\
\text { experiência em emergência; } \\
\text { (3) falhas na comunicação } \\
\text { - falta de informações na } \\
\text { admissão do paciente e } \\
\text { passagem de casos; (4) } \\
\text { falhas organizacionais - falta } \\
\text { de leitos, recursos humanos } \\
\text { inadequados, delimitação } \\
\text { de responsabilidades no } \\
\text { processo de assistência } \\
\text { e falhas de registros em } \\
\text { prontuário eletrônico. }\end{array}$ & $\begin{array}{l}\text { A segurança } \\
\text { depende de um } \\
\text { sistema complexo } \\
\text { e multifatorial } \\
\text { que propicia } \\
\text { riscos para o } \\
\text { paciente nas } \\
\text { unidades de } \\
\text { emergência. A } \\
\text { relação entre os } \\
\text { fatores de risco é } \\
\text { pouco conhecida } \\
\text { e necessita } \\
\text { ser explorada } \\
\text { em pesquisas } \\
\text { com foco na } \\
\text { interação entre } \\
\text { processos clínicos, } \\
\text { especialmente } \\
\text { com a alta carga } \\
\text { de trabalho. }\end{array}$ \\
\hline
\end{tabular}

Fonte: Elaboração própria.

\section{Discussão}

Foram apreendidas três categorias: Fatores Organizacionais, Falhas na Comunicação da Equipe e Fragilidades no Processo de Medicação.

\section{Fatores Organizacionais}

Esta categoria originou-se em seis estudos ${ }^{(19-20,23,25-27)}$ que abordam a interferência do ambiente de trabalho na segurança da assistência, expressa pela dinâmica agitada do trabalho e escassez de normatizações institucionais/assistenciais, as quais dificultam a gestão dos serviços de emergência. Um dos estudos destaca a necessidade de otimização das metodologias de pesquisas que elucidem outras características do trabalho de Unidades de Emergência (UE), como alta carga de trabalho e interrupções durante a execução do cuidado, as quais impactam na segurança do paciente ${ }^{(20)}$. Também são destacadas estratégias para compreensão do comportamento humano em sistemas organizacionais e desenvolvimento de métodos que revelem condições latentes ao erro ${ }^{(20)}$.

O estudo realizado na Austrália ${ }^{(26)}$ constatou alto nível de exaustão emocional em profissionais de UE relacionada ao ambiente contextual de atendimento e demanda excessiva de pacientes. Essa é uma realidade vivenciada em estudos de outros países, que também apontam a falta de especialistas nos plantões de emergência, diversidade e condições clínicas complexas dos pacientes, escassez de leitos para internação hospitalar e estresse ocupacional da equipe $^{(28)}$. Em estudo analisado ${ }^{(27)}$, falhas organizacionais mostraram relação com a falta de leitos para internação e o nível de pessoal insuficiente, fatores que resultaram, respectivamente, no não recebimento dos cuidados necessários, atrasos de alta e elevação do tempo de espera para a primeira avaliação.

Ainda sobre os fatores presentes no ambiente de UE, pesquisa realizada na Bélgica indicou o 
número elevado de pacientes presentes no setor como indicador moderado de insegurança na assistência. Considerou-se que a superlotação acarretou em tempo de espera inseguro, isto é, implicou em demora na definição e realização de condutas terapêuticas ${ }^{(20)}$. De acordo com o Emergency Severity Index (ESI), o tempo de espera inseguro ocorre quando pacientes que necessitam de intervenções imediatas aguardam por mais de 10 minutos para o atendimento nas UE ou 30 minutos para pacientes que apresentam condições de alto risco, como, por exemplo, dor torácica ativa, suspeita de doença coronariana aguda, sinais de acidente vascular cerebral, imunossuprimido com febre ou dor/dificuldade severa.

Uma revisão de literatura retratou que o tempo de espera inseguro ocasiona problemas como: resultados desfavoráveis ao tratamento e à evolução do paciente, maior sofrimento para os familiares, alta tensão na equipe assistencial e ambiente propenso a estresse no trabalho. Esse é um problema a ser resolvido com urgência, porque gera atraso no diagnóstico e no tratamento, fatores que, consequentemente, podem levar ao aumento da mortalidade ${ }^{(29)}$. Ainda no campo organizacional, uma pesquisa nacional acerca da organização do trabalho da enfermagem cita alguns instrumentos que podem ser utilizados pelo serviço para manter a organização do setor como: regulamentos, organograma, sistematização de técnicas, rotinas e sistemas de controle, além de atividades de educação permanente para a equipe ${ }^{(30)}$.

O ritmo intenso de trabalho nos serviços de emergência torna os profissionais suscetíveis às falhas excessivas durante a execução de suas tarefas, circunstância que pode impactar negativamente nos resultados assistenciais ${ }^{(11)}$. Outras pesquisas $^{(19,31)}$ apontam a ausência de planejamento organizacional e troca de informações acerca do paciente durante a execução do cuidado, como fatores que desviam a atenção do profissional no trabalho. Considerando tais fatores, um dos artigos analisados nesta revisão ${ }^{(25)}$ sugere que interrupções desnecessárias comprometem a organização da assistência, por facilitar a ocorrência de incidentes no processo de administração de medicamentos, como erros na administração e reações adversas.

No cenário brasileiro, pesquisa transversal realizada na região do Distrito Federal, com foco nos erros de medicação, também evidenciou que interrupções durante a execução do cuidado e número elevado de pacientes durante o turno de trabalho são fatores que contribuem para o aumento do risco de erro ${ }^{(32)}$. Para minimizar as interrupções, os autores sugerem a identificação de restrições de trabalho e a necessidade de desenvolver intervenções para gerenciar interrupções, sendo essencial que essas tenham um impacto negativo mínimo no desempenho ${ }^{(33)}$.

\section{Falhas na Comunicação da Equipe}

Nesta categoria, quatro estudos ${ }^{(21,23,26-27)}$ retrataram as falhas de comunicação entre profissionais como um dos fatores que interferiam negativamente na segurança do paciente, por serem contribuintes comuns a erros nos cuidados de saúde. Ao gerar lacunas no compartilhamento de informações, a continuidade da assistência pode ser comprometida, principalmente em etapas de transição do cuidado, considerando que, se informações relevantes para a oferta de cuidado são desconhecidas da equipe, o paciente pode ser exposto a situação de risco, como, por exemplo, administração de medicamento alérgeno.

Evidências da literatura apontam a falta de comunicação ou comunicação inapropriada entre a equipe multiprofissional, como aspectos que interferem na fluidez do processo de trabalho e prejudicam a construção de metas e objetivos comuns ${ }^{(6)}$.

Um dos estudos analisados ${ }^{(23)}$ foi realizado com o objetivo de explorar como os profissionais da saúde, os pacientes e os membros da família comunicam-se. Constatou-se que a comunicação efetiva entre profissional, paciente e/ou acompanhante também é indispensável no processo do cuidar em urgência e emergência, uma vez que informações sobre o histórico de saúde contribuem para o manejo clínico, além 
de possibilitar ações pró-ativas nos pacientes e/ou acompanhantes.

Tal achado confirma pesquisa realizada no Brasil, com o objetivo de apreender a opinião de médicos atuantes em UE, na qual discutiu-se a importância da comunicação médico-paciente. Os autores afirmaram que falta de informações acerca do paciente repercutia negativamente no processo de decisão clínica, devido à falta de acesso a dados importantes sobre a saúde e a doença do paciente ${ }^{(34)}$.

Ainda no que se refere à comunicação como elemento da segurança do paciente, as etapas detransição dos cuidados em saúde necessitam de rotinas gerenciais relacionadas à transmissão de informações, como a passagem de plantão. Sabe-se que, em UE, a rotina de troca de plantões também sofre influência das peculiaridades desse ambiente de trabalho e, mesmo com a flexibilidade apresentada por uma equipe adaptada às rotinas, o processo de trabalho dinâmico e inconstante e a alta demanda de pacientes comprometem a qualidade do processo conhecido como handoff ${ }^{(35)}$.

O handoff, que consiste no momento do processo de trabalho no qual informações clínicas são repassadas entre profissionais de funções e/ou setores diferentes, é uma importante ferramenta no gerenciamento dos riscos asssistenciais, pois proporciona aos profissionais a oportunidade de transmitir diretamente informações acerca do paciente que garantirão a qualidade e a segurança da assistência ${ }^{(33)}$. Contudo, a literatura $^{(27)}$ aponta falhas processuais no handoff caracterizadas por interrupções frequentes que implicam na perda e/ou interpretação equivocada das informações.

Uma recente revisão da literatura ${ }^{(36)}$ sintetizou dados referentes às ações gerenciais e assistenciais para a promoção da segurança do paciente em serviços hospitalares e evidenciou predomínio de estratégias para notificação de erros e escassez de ações voltadas à melhoria da comunicação entre os membros da equipe de saúde.

Assim, embora o trabalho em saúde seja essencialmente relacional, a comunicação ainda se apresenta frágil. Além disso, este estudo identificou o processo de medicação como potencial determinante para a ocorrência de eventos adversos ao paciente.

\section{Falhas no Processo de Medicação}

A categoria surgiu em três estudos da amostra $^{(21,24,26)}$ caracterizada pela prescrição incorreta dos medicamentos, ausência de anotações, registros incorretos e checagem inadequada de medicamentos. Nesse aspecto, um estudo desta revisão ${ }^{(26)}$ alerta que os erros de medicação tendem a ser mais frequentes em UE devido à dinâmica agitada de trabalho e à ineficácia dos sistemas de informação, que apresentam dados incompletos acerca do paciente. Todavia, na atenção em saúde, sabe-se que os erros identificados não ocorrem de forma isolada. Com isso, tanto a instituição como os profissionais devem estar diretamente envolvidos na descoberta de condições latentes que favorecem a ocorrência de eventos adversos no cuidado ${ }^{(14)}$.

Os resultados obtidos em um dos estudos analisados ${ }^{(22)}$ enfatiza os erros relacionados à administração de medicamentos e ausência de checagem de medicamentos pela enfermagem. Em outra publicação $^{(37)}$, os tipos de erros mais frequentes no processo de medição referem-se à prescrição e administração, como droga errada/não autorizada, dose errada, dose extra, droga preparada incorretamente, técnica de administração errada, paciente errado e via errada. De acordo com os autores, tais erros resultam do déficit de desempenho, procedimento ou protocolo não seguido adequamente, déficit de conhecimento, comunicação não efetiva, prescrição verbal, erros de cálculo e monitoramento inadequado.

Ainda sobre as causas que geram erros de medicação, um dos artigos selecionados nesta revisão ${ }^{(22)}$ aponta idade do paciente, prescrição de mais de um medicamento e prescrição realizada por residentes como fatores associados aos erros nas prescrições médicas. Nessa mesma direção, pesquisa realizada no ano de $1998^{(38)}$ em um hospital terciário dos Estados Unidos, já havia constatado que residentes do primeiro ano de medicina apresentavam maiores taxas de erros de prescrição do que os residentes com maior tempo de atuação. Esses achados sugerem 
que a presença de estagiários e/ou a pouca experência no serviço explicam, em parte, a maior taxa de erros no Departamento de Emergência.

A literatura inserida nessa categoria ${ }^{(22)}$ expõe que a idade, em especial os idosos, tende a ter mais comorbidades e maior número de medicamentos, os quais podem resultar em contra-indicações e interações, o que complica o processo de prescrição. Em situações de mais de um medicamento prescrito, a complexidade da prescrição aumenta à medida que é maior o número de medicamentos, ampliando, assim, o potencial de erro. Outra análise transversal e descritiva realizada em um Hospital Universitário menciona que o maior número de erros foram encontrados nas prescrições de residentes em medicina de emergência, por terem menos experiência com a amplitude dos serviços da UE e por estarem menos familiarizados com medicamentos, dosagens, regimes de dosagem e tratamentos ${ }^{(39)}$.

Além de erros na prescrição de medicamentos, a administração e os registros relacionados a essa etapa também podem representar risco ao paciente, quando não são realizados adequadamente. Estudo descritivo, retrospectivo, desenvolvido no Pronto Socorro de um Hospital Universitário no Brasil $^{(24)}$, o qual faz parte da amostra incluída nesta revisão, aponta a ausência de anotações e a checagem inadequada de medicamentos como omissão de cuidado. Ainda sobre essa problemática, estudo descritivo exploratório, realizada na Austrália, relata que falhas de medicação ocorrem com mais freqüência na UE, devido ao número elevado e/ou à falta de identificação dos pacientes, estado de alergia e erros de omissão, aliados ao déficit de trabalhadores da enfermagem ${ }^{(14)}$.

Em dois estudos analisados ${ }^{(20,24)}$, para mitigação dos riscos referentes aos erros de medicação, os autores recomendaram a abordagem sistêmica do erro, pois pode revelar falhas no processo. Acrescentam ainda que os farmacêuticos podem desempenhar papel importante na identificação e diminuição dos erros de prescrição. Outro estudo ${ }^{(20)}$ menciona que o trabalho interdisciplinar com os serviços disponíveis no setor da farmácia reduzem os danos associados aos erros. Logo, o processo de medicação do setor de emergência abrage peculiaridades que podem levar à ocorrência de erros. Estes, por sua vez, tendem a impactar na segurança do paciente e na qualidade do cuidado ${ }^{(40)}$.

O número reduzido de publicações com o foco nos serviços de urgência e emergência foi uma limitação apresentada pelo estudo. Desta forma, a pesquisa realizada constituiu-se em uma importante lacuna identificada e explorada.

\section{Conclusão}

A comunicação entre a equipe e a gestão de medicamentos são fatores que induzem aos erros em UE. Desta forma, é necessário criar estratégias voltadas à compreensão do comportamento humano em sistemas organizacionais, bem como o desenvolvimento de métodos e/ou instrumentos que revelem as condições latentes ao erro nesses espaços.

Os resultados desta revisão integrativa contribuem para a prática assistencial, ao indicarem fatores que interferem na prestação de cuidado seguro, o que pode sensibilizar gestores de instituições de saúde quanto à segurança do paciente em ambiente de urgência e emergência, e subsidiar o planejamento de estratégias de mitigação de riscos e de promoção de segurança nesses espaços. No âmbito da pesquisa, este estudo destaca os fatores de insegurança presentes na literatura e indica que ainda é necessário ampliar a investigação sobre este tema dada a sua complexidade e as particularidades do ambiente de UE. Além disso, quanto ao ensino, esta pesquisa contribui para o debate sobre segurança do paciente desde a formação, considerando que o tema é de grande relevância para o panorama de saúde nacional e internacional.

\section{Colaborações:}

1 - concepção, projeto, análise e interpretação dos dados: Eloyne Tavares da Silva, Laura Misue Matsuda, Gabriela Machado Ezaias Paulino, Nadia Raquel Suzini Camillo, Ana Carolina Simões e Andressa Martins Dias Ferreira; 
2 - redação do artigo e revisão crítica relevante do conteúdo intelectual: Eloyne Tavares da Silva, Laura Misue Matsuda, Gabriela Machado Ezaias Paulino e Andressa Martins Dias Ferreira;

3 - aprovação final da versão a ser publicada: Eloyne Tavares da Silva, Laura Misue Matsuda, Gabriela Machado Ezaias Paulino e Andressa Martins Dias Ferreira.

\section{Referências}

1. Fonseca CN, Guerra TRB, Dehoul MS, Souto Maior MCG. Segurança do paciente em uma grande emergência do SUS: como assegurar a prática? Academus Rev Cient Saúde [Internet]. 2016 [cited 2018 Jun 15];1(2). Available from: https://smsrio.org/revista/index.php/reva/article/ view/144

2. Silva AJ, Pinto MC. Avaliando o impacto da estratégia de segurança do paciente implantada em uma unidade de clínica médica de um hospital universitário sob a perspectiva da dimensão da atenção à saúde. Rev Adm Saúde. 2017;17(66):1-15. DOI: http://dx.doi.org/10.23973/ras.66.10

3. Brasil. Ministério da Saúde. Documento de referência para o Programa Nacional de Segurança do Paciente. Brasília (DF); 2014. Disponível em: http://bvsms.saude.gov.br/bvs/publicacoes/ documento_referencia_programa_nacional_ seguranca.pdf.

4. Kohn LT, Corrigan JM, Donaldson MS. To Err Is Human: Building a Safer Health System. Washington (DC): National Academies Press; 2000.

5. Lorenzini E, Santi JAR, Baó ACP. Segurança do paciente: análise dos incidentes notificados em um hospital do Sul do Brasil. Rev Gaúcha Enferm. 2014;35(2):121-7. DOI: http://dx.doi. org/10.1590/1983-1447.2014.02.44370

6. Duarte MCS, Queiroz ABA, Büscher A, Stipp CAM. O erro humano no cotidiano da assistência de enfermagem em terapia intensiva. Rev Latino-Am Enfermagem. 2015;23(6):1074-81. DOI: http:// dx.doi.org/10.1590/0104-1169.0479.2651

7. Brixey JJ, Robinson DJ, Johnson CW, Johnson TR, Turley JP, Patel VL, et al. Towards a hybrid method to categorize interruption and activities in health care. Int J Med Inform. 2007;76(11-12):812-20. DOI: http://dx.doi. org/10.1016/j.ijmedinf.2006.09.018
8. Laxmisan A, Hakimzada F, Sayan OR, GreenAR,ZhangJ,PatelV. Themultitaskingclinician: decision-making and cognitive demand during and after team handoffs in emergency care. Int $\mathrm{J}$ Med Inform. 2007 Nov-Dec;76(11-12):801-11. DOI: http://dx.doi.org/10.1016/j.ijmedinf.2006.09.019

9. Bernstein SL, Aronsky D, Duseja R, Epstein S, Handel D, Hwang U, et al. The effect of emergency department crowding on clinically oriented out comes. Acad Emerg Med. 2009 Jan;16(1):1-10. DOI: http://dx.doi. org/10.1111/j.1553-2712.2008.00295.x.

10. Cielo C, Camponogara S, Pillon FBR. A comunicação no cuidado à saúde em unidade de urgência e emergência: relato de experiência. J Nurs Health. 2013;3(2):204-12. DOI: http://dx.doi.org/10.15210/ jonah.v3i2.3398

11. Crossetti OGM, Bittencourt DGKG, Lima AAA, Góes MGO, Saurin G. Elementos estruturais do pensamento crítico de enfermeiros atuantes em emergências. Rev Gaúcha Enferm. 2014;35(3):55-60. DOI: http://dx.doi.org/10.1590/1983-1447.2014.03.45947

12. Rossetti CA, Gaidzinski RR, Bracco MM. Determinação da carga de trabalho e do dimensionamento da equipe de enfermagem em um pronto-socorro pediátrico. Einstein. 2014;12(2):217-22. DOI: http://dx.doi.org/10.1590/ S1679-45082014AO2945

13. Gallotti RMD. Eventos adversos: o que são? Rev Assoc Méd Bras. 2004;50(2):109-26. DOI: http:// dx.doi.org/10.1590/S0104-42302004000200008

14. Souza TM, Silva DM, Carvalho R. Revisão integrativa: o que é e como fazer? Einstein. 2010 jan-mar;8(1):102-6. DOI: http://dx.doi.org/10.1590/ s1679-45082010rw1134

15. Mendes KDS, Silveira RCCP, Galvão CM. Revisão integrativa: método de pesquisa para a incorporação de evidências na saúde e na enfermagem. Texto Contexto Enferm. 2008;17(4):758-64. DOI: http:// dx.doi.org/10.1590/S0104-07072008000400018

16. Stillwell SB, Fineout-Overholt E, Melnyk BM, Williamson KM. Evidence-based practice, step by step: asking the clinical question: a key step in evidence-based pratice. Am J Nurs. 2010 Mar;110(3):58-61. DOI:10.1097/01. naj.0000368959.11129.79

17. Caldwell K, Henshaw L, Taylor G. Developing a framework for critiquing health research: an early evaluation. Nurse educ today. 2011 Nov;31(8):e1-e7. DOI: http://dx.doi.org/10.1016/j. nedt.2010.11.025 
18. Ursi ES, Galvão CM. Prevençãoo de lesões de pele no perioperatório: revisão integrativa da literatura. Rev Latino-Am Enfermagem. 2006;14(1):124-31. DOI: http://dx.doi.org/10.1590/ S0104-11692006000100017

19. Bardin L. Análise de conteúdo. Lisboa: Edições 70; 2011.

20. Bergs J, Verelst S, Gillet BJ, Deboutte P, Vandoren C, Vandijck D. The number of patients simultaneously present at the emergency department as an indicator of unsafe waiting times: a receiver operated curve-based evaluation. Int Emerg Nurs. 2014;22(4):185-9. DOI: http://dx.doi.org/10.1016/j. ienj.2014.01.002

21. Källberg SA, Göransson EK, Florin J, Östergren J, Brixey JJ, Ehrenberg A. Contributing factors to errors in Swedish emergency departments. Int Emerg Nurs. 2015;23(2):156-61. DOI: http:// dx.doi.org/10.1016/j.ienj.2014.10.002

22. Stasiak P, Afilalo M, Castelino T, Xue X, Colacone A, Soucy N, et al. Detection and correction of prescription erros by an emergency department pharmacy service. CJEM. 2014 May;16(3):193-206. DOI: $10.2310 / 8000.2013 .130975$

23. Manias E, Gerdtz M, Williams A, Dooley M. Complexities of medicines safety: communicating about managing medicines at transition points of care across emergency departments and medical wards. J Clin Nurs. 2014 Jan;24(1-2):69-80. DOI: http://dx.doi.org/10.1111/jocn.12685

24. Paranaguá TTB, Bezerra ALQ, Santos ALM, Silva AEBC. Incidentes: instrumento de gerenciamento da assistência para a segurança do paciente em pronto socorro. Enferm Global [Internet]. 2014 [cited 2018 May 15];(34):219-31. Available from: http://scielo.isciii.es/pdf/eg/v13n34/pt_ administracion1.pdf

25. Alcaraz-Martínez J, Aranaz-Andrés JM, Martínez-Ros C, Moreno-Reina S, Escobar-Álvaro L, Ortega-Liartef JV,et al. Estudio Regional de Incidentes Derivados de la Atención (ERIDA) em Urgencias. Rev Calid Asist. 2017;32(1):285-92. DOI: https://doi.org/10.1016/j.cali.2015.12.011

26. Weigl M, Schneider A. Associations of work characteristics, employee strain and self-perceived quality of care in Emergency Departments: A cross-sectional study. Int Emerg Nurs. 2017;30:2024. DOI: https://doi.org/10.1016/j.ienj.2016.07.002

27. Källberg AS, Ehrenberg A, Florin J, Östergren J, Göransson KE. Physicians' and nurses' perceptions of patient safety risks in the emergency department. Int Emerg Nurs. 2017;33:14-19. DOI: 10.1016/j. ienj.2017.01.002

28. Pham CJ, Alblaihed L, Cheung SD, Levy F, Hill MP, Kelen DG, et al. Measuring Patient Safety in the Emergency Department. Am J Med Qual. 2014 Mar-Apr;29(2);99-104, DOI: https://doi. org/10.1177/1062860613489846

29. Bittencourt RJ, Hortale VA. Intervenções para a superlotação nos serviços de emergência hospitalar; uma revisão sistemática. Cad Saúde Pública. 2009;25(7):1439-54. DOI: http://dx.doi. org/10.1590/S0102-311X2009000700002

30. Lima SBS, Erdmann AL. A enfermagem no processo da acreditação hospitalar em um serviço de urgência e emergência. Acta Paul Enferm. 2006;19(3):271-8. DOI: http://dx.doi.org/10.1590/ S0103-21002006000300003

31. Johnston A, Abraham L, Greenslade J, Thom O, Carlstrom E, Wallis M, et al. Review article: Staff perception of the emergency department working environment: Integrative review of the literature. Emerg Med Australas. 2016 Feb;28(1):7-26. DOI: https://doi.org/10.1111/1742-6723.12522

32. Volpe CRG, Melo EMM, Aguiar LB, Pinho DLM, Stival MM. Risk factors for medication errors in the electronic and manual prescription. Rev Latino-Am Enfermagem. 2016 Aug;24:e2742. DOI: https://doi. org/10.1590/1518-8345.0642.2742

33. Antonelli RC, Bellucci Junior JA. Gerenciamento de enfermagem em serviço hospitalar de emergência: revisão integrativa da literatura. Semina ciênc biol saude. 2014 jul/dez;35(2):137-46. DOI: https://doi. org/10.5433/1679-0367.2014v35n2p137

34. Farley HL, Baumlin KM, Hamedani AG, Cheung DS, Edwards MR, Fuller DC, et al. Quality and safety implications of emergency department information systems. Ann Emerg Med. 2013;62(4):399-407. DOI: https://doi. org/10.1016/j.annemergmed.2013.05.019

35. Frush K. Whyandwhento use CT in children: perspective of a pediatric emergency medicine physician. Pediatr Radiol. 2014;44(Suppl 3):409-13. DOI: https://doi.org/10.1007/s00247-014-3122-x

36. Siman AG, Cunha SGS, Brito MJM. Ações de enfermagem para segurança do paciente em hospitais: revisão integrativa. Rev enferm UFPE. 2017;11(Supl 2):1016-24. DOI: https:// doi.org/10.5205/reuol.10263-91568-1-RV. 1102sup201718 
Eloyne Tavares da Silva, Laura Misue Matsuda, Gabriela Machado Ezaias Paulino, Nadia Raquel Suzini Camillo, Ana Carolina Simões, Andressa Martins Dias Ferreira

37. Pham CJ, Andrawis M, Shore DA, Fahey M, Morlock L, Pronovost PJ. Are temporary staff associated with more severe emergency department medication errors? J Healthc Qual. 2011 Jul-Aug;33(4):9-18. DOI: https://doi. org/10.1111/j.1945-1474.2010.00116.x

38. Lesar TS. Errors in the use of drug dosage equations. Arch Pediatr Adolesc Med. 1998;152(4):340-4. DOI: https://doi.org/10.1001/archpedi.152.4.340

39. Velho MTAC, Haeffner LB, Santos FG, Silva LC, Weinmann ARM. Residência médica em um hospital universitário: a visão dos residentes. Rev
Bras Educ Méd. 2012;36(3):351-7. DOI: http:// dx.doi.org/10.1590/S0100-55022012000500009

40. Oliveira RC, Camargo AEB, Cassiani SHDB. Estratégias para prevenção de erros na medicação no setor de mergência. Rev Bras Enferm. 2005;58(4):399-404. DOI: 10.1590/ s0034-71672005000400004

Recebido: 13 de outubro de 2019

Aprovado: 12 de novembro de 2019

Publicado: 16 de março de 2020

A Revista Baiana de Enfermagem utiliza a Licença Creative Commons - Atribuição-NãoComercial 4.0 Internacional. https://creativecommons.org/licenses/by-nc/4.0/

Este artigo é de acesso aberto distribuído sob os termos da Licença Creative Commons (CC BY-NC).

Esta licença permite que outros remixem, adaptem e criem a partir do seu trabalho para fins não comerciais. Embora os novos trabalhos tenham de lhe atribuir o devido crédito e não possam ser usados para fins comerciais, os usuários não têm de licenciar esses trabalhos derivados sob os mesmos termos. 